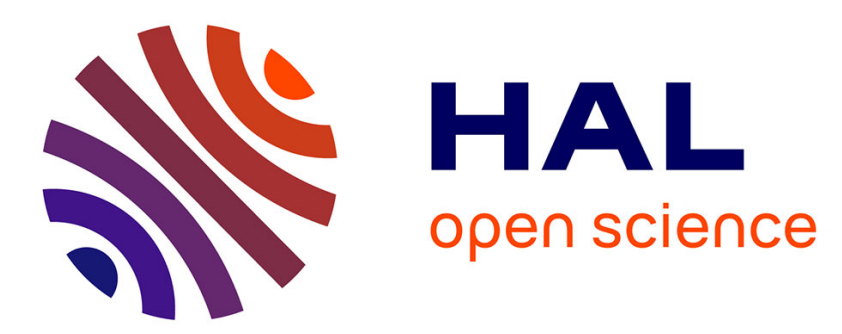

\title{
Orthogonal decomposition of derivatives and antiderivatives for easy evaluation of extended Gram matrix
}

Mihai Telescu, Pascale Bréhonnet, Noël Tanguy, Pierre Vilbé, Léon-Claude Calvez

\section{To cite this version:}

Mihai Telescu, Pascale Bréhonnet, Noël Tanguy, Pierre Vilbé, Léon-Claude Calvez. Orthogonal decomposition of derivatives and antiderivatives for easy evaluation of extended Gram matrix. Signal Processing, 2006, 86 (11), pp.3486-3489. 10.1016/j.sigpro.2006.06.007 . hal-00174253

\section{HAL Id: hal-00174253 \\ https://hal.science/hal-00174253}

Submitted on 21 Feb 2014

HAL is a multi-disciplinary open access archive for the deposit and dissemination of scientific research documents, whether they are published or not. The documents may come from teaching and research institutions in France or abroad, or from public or private research centers.
L'archive ouverte pluridisciplinaire HAL, est destinée au dépôt et à la diffusion de documents scientifiques de niveau recherche, publiés ou non, émanant des établissements d'enseignement et de recherche français ou étrangers, des laboratoires publics ou privés. 


\title{
Orthogonal decomposition of derivatives and antiderivatives for easy evaluation of extended Gram matrix
}

\author{
M. Telescu, P. Bréhonnet, N. Tanguy, P. Vilbé, and L.C. Calvez \\ Laboratoire d'Electronique et Systèmes de Télécommunications (LEST), \\ UMR CNRS 6165 - site Université de Bretagne Occidentale (UBO), \\ 6, av. Le Gorgeu, CS 93837, 29238 BREST Cedex 3, France
}

Keywords: Orthogonal representation, Gram matrix, Routh algorithm, Model-order reduction

\begin{abstract}
Simple and efficient algorithms for orthogonal decomposition of derivatives and antiderivatives of a function with rational Laplace transform are proposed. Based on a new theorem related to Routh $\alpha-\beta$ expansion, they enable direct evaluation of the extended Gram matrix which has proved to be very useful in model-reduction applications.
\end{abstract}

\section{1 - Introduction}

Since the pioneering work of Jain $[1,2]$ the Gram matrix has proved to be one of the most reliable tools in the field of model reduction [3-6]; its ability to produce good pole positions has motivated a number of papers. Early work has been devoted to the Gram matrix of successive antiderivatives of a time response. Some of the authors [3] have shown that evaluation of this Gram matrix can be achieved in the frequency domain; in a sequel to [3], Lucas [7] and Sreeram and Goddard [8] presented additional simplifications in computation. Sreeram and Agathoklis [5,9] have shown the connection between the Gram matrix and the other Gramians, whereby the Gram matrix can be obtained by solving Lyapunov equations.

In $[4,6]$ the Gram matrix has been successfully extended to include both derivatives and antiderivatives. It is the purpose of this communication to propose a straightforward orthogonal decomposition of these derivatives and antiderivatives, directly obtained from the so-called Routh $\alpha-\beta$ parameters. Owing to this orthogonal representation, an elementary and simple computation of the extended Gram matrix follows readily

\section{2 - Background}

Although the Routh $\alpha-\beta$ tables are familiar to model reduction researchers, to make this paper self contained and to state precisely some notations and numberings, the procedure is first outlined below in polynomial form; for a tabular form see [10].

Let $D(s)=\sum_{i=0}^{n} d_{i} s^{i}$ denote a strictly Hurwitz polynomial. Starting with its even and odd parts, $D_{n}(s)=d_{n} s^{n}+d_{n-2} s^{n-2}+\ldots$ and $D_{n-1}(s)=d_{n-1} s^{n-1}+d_{n-3} s^{n-3}+\ldots$, let a sequence of polynomials of descending degree be computed recursively by the formula

$$
D_{k-1}=D_{k+1}-\alpha_{k} s D_{k}, \quad k=n-1, \ldots, 1
$$

with $\alpha_{k}=l c\left(D_{k+1}\right) / l c\left(D_{k}\right), \quad k=n-1, \ldots, 0$, where $l c(P)$ denotes the leading coefficient of the polynomial $P$. Notice that the numbering as defined in [10] has been modified for convenience. A strictly proper rational Laplace transform $F(s)=N(s) / D(s)$ that is asymptotically stable can always be decomposed into the following form 


$$
F(s)=\sum_{k=0}^{n-1} \beta_{k} D_{k}(s) / D(s)
$$

in which the coefficients $\beta_{k}$ are uniquely determined by $N(s)=\sum_{k=0}^{n-1} \beta_{k} D_{k}(s)$. It is known [11] that the Laplace transforms $\Phi_{k}(s) \hat{=} D_{k}(s) / D(s), \quad k=0, \ldots, n-1$, define a set of $n$ orthogonal time functions $\varphi_{k}(t)$ :

$$
\left\langle\varphi_{k}, \varphi_{i}\right\rangle \hat{=} \int_{0}^{\infty} \varphi_{k}(t) \varphi_{i}(t) d t=\delta_{k i} /\left(2 \alpha_{k}\right)
$$

As far as the authors are aware, the following theorem, which is the keystone of the two algorithms to be proposed in this paper, has never been mentioned before.

Theorem 1: Define $\varphi_{-1} \hat{=} 0$ and $\varphi_{n} \hat{=}-\varphi_{n-1}$. Then the derivative of $\varphi_{k}(t)$ satisfies

$$
\alpha_{k} \frac{d \varphi_{k}(t)}{d t}=\varphi_{k+1}(t)-\varphi_{k-1}(t), k=0, \ldots, n-1
$$

Proof of Theorem 1: On the understanding that $D_{-1}=0$, eqn. 1 can be rewritten as

$$
s D_{k}=\left(D_{k+1}-D_{k-1}\right) / \alpha_{k}, k=0, \ldots, n-1
$$

Assume $k \leq n-2$. The initial value theorem for the Laplace transform yields $\varphi_{k}(+0)=0$, therefore the Laplace transform of $d \varphi_{k}(t) / d t$ is $s D_{k} / D$. Thus, starting from eqn. 5 and dividing throughout by $D$, eqn. 4 is proved for $k=0, \ldots, n-2$. Now, consider $\varphi_{n-1}(+0)=d_{n-1} / d_{n}=1 / \alpha_{n-1}$, hence the Laplace transform of $d \varphi_{n-1}(t) / d t$ is $\left(s D_{n-1} / D_{n}\right)-1 / \alpha_{n-1}$ which, on account of eqn. 5 and $D_{n}+D_{n-1}=D$ may be written $-\left(D_{n-1}+D_{n-2}\right) /\left(\alpha_{n-1} D\right)$. This achieves the proof of eqn. 4 for $k=n-1$.

An extended Gram matrix involves inner products of signals $f_{i}(t), \quad i \in Z$, recursively defined by $f_{0}(t) \hat{=} f(t), f_{i+1}(t) \hat{=} d f_{i}(t) / d t$ and $f_{i-1}(t) \hat{=} \int_{\infty}^{t} f_{i}(\tau) d \tau$. Applying the inverse Laplace transform to eqn. 2 yields the orthogonal decomposition $f_{0}(t)=\sum_{k=0}^{n-1} \beta_{k} \varphi_{k}(t)$. More generally, owing to the pole preserving property of operators $d / d t$ and $\int_{\infty}^{t}$, any $f_{i}(t)$ admits an orthogonal decomposition of the following form

$$
f_{i}(t)=\sum_{k=0}^{n-1} \beta_{k}^{i} \varphi_{k}(t)
$$

Starting from $\beta_{k}^{0} \hat{=} \beta_{k}$, the coefficients $\beta_{k}^{i}$ can be efficiently computed for $i=1,2, \ldots$ and $i=-1,-2, \ldots$ by algorithms $D^{+}$and $D^{-}$proposed below.

Algorithm $D^{+}$: Given $\alpha_{k}, \beta_{k}^{i}$ and $\theta_{k}^{i} \hat{=} \beta_{k}^{i} / \alpha_{k}$ relative to $f_{i}(t)$, let $\theta_{-1}^{i} \hat{=} 0$ and $\theta_{n}^{i} \hat{=} \theta_{n-1}^{i}$; then the following algorithm computes the $\beta$ 's and $\theta$ 's relative to the derivative $f_{i+1}(t)$ :

$$
\begin{aligned}
& \text { For } \begin{aligned}
& k= 0, \ldots, n-1 \\
& \beta_{k}^{i+1}:=\theta_{k-1}^{i}-\theta_{k+1}^{i} \\
& \theta_{k}^{i+1}:=\beta_{k}^{i+1} / \alpha_{k}
\end{aligned}
\end{aligned}
$$

Proof of Algorithm $D^{+}$: Differentiating eqn. 6 with respect to $t$, using eqn. 4 and rearranging yields $f_{i+1}(t)=\sum_{k=0}^{n-1}\left(\theta_{k-1}^{i}-\theta_{k+1}^{i}\right) \varphi_{k}(t)$ which achieves the proof. This algorithm takes only $(n-1)$ additive operations $(+,-)$ and $n$ multiplicative operations $(\times, \div)$. For comparison, the standard way to compute $\beta_{k}^{i+1}$ is to evaluate the Laplace transform of $f_{i+1}(t)$ from that of $f_{i}(t)$ and then to construct the related $\beta$-table. The first step takes exactly the same number of operations as algorithm $D^{+}$. Hence, the orthogonal decomposition of $f_{i+1}(t)$ via algorithm $D^{+}$can be obtained as cheaply as its Laplace transform. 
In short, algorithm $D^{+}$saves the $\left\lceil\frac{n}{2}\right]\left(\left\lfloor\frac{n}{2}\right\rfloor+1\right)$ multiplicative operations and $\left\lfloor\frac{n}{2}\right\rfloor\left(\left\lceil\frac{n}{2}\right\rceil-1\right)$ additive operations required by the $\beta$-table, where $\lfloor x\rfloor$ denotes the integer part of $x$ and $\lceil x\rceil$ denotes the smallest integer greater than or equal to $x$.

Algorithm $D^{-}$: Given $\alpha_{k}, \beta_{k}^{i}$ and $\theta_{k}^{i} \hat{=} \beta_{k}^{i} / \alpha_{k}$ relative to $f_{i}(t)$, define $\theta_{-1}^{i-1} \hat{=} 0$; then the following algorithm computes $\beta$ 's and $\theta$ 's relative to the antiderivative $f_{i-1}(t)$ :

$$
\begin{aligned}
& \text { For } \begin{aligned}
k= & 0, \ldots,\left\lfloor\frac{n-2}{2}\right\rfloor \\
& \theta_{2 k+1}^{i-1}:=\theta_{2 k-1}^{i-1}-\beta_{2 k}^{i} \\
& \text { if } n \text { is odd then } \theta_{n-1}^{i-1}:=\theta_{n-2}^{i-1}-\beta_{n-1}^{i} \\
& \text { else } \theta_{n-2}^{i-1}:=\theta_{n-1}^{i-1}+\beta_{n-1}^{i}
\end{aligned} \\
& \text { For } k=\left\lfloor\frac{n-1}{2}\right\rfloor, \ldots, 1 \\
& \text { For } k=0, \ldots, n-1 \\
& \quad \theta_{2 k-2}^{i-1}:=\theta_{2 k}^{i-1}+\beta_{2 k-1}^{i} \\
& \quad \beta_{k}^{i-1}:=\alpha_{k} \theta_{k}^{i-1}
\end{aligned}
$$

Proof of Algorithm $D^{-}$: Replacing $i$ by $i-1$ in algorithm $D^{+}$we have $\beta_{k}^{i}=\theta_{k-1}^{i-1}-\theta_{k+1}^{i-1}, \theta_{k}^{i}=\beta_{k}^{i} / \alpha_{k}$. Letting $k=0$, with $\theta_{-1}^{i-1}=0$ in mind, yields $\theta_{1}^{i-1}=-\beta_{0}^{i}$; then it is a simple matter to see that the $\theta_{k}^{i-1}$ can be computed in succession, in the order indicated by algorithm $D^{-}$. Once again the orthogonal decomposition is obtained as cheaply as the Laplace transform and all the operations required by the $\beta$-table are saved.

Theorem 2: Let $B=\left[b_{i j}\right]$ and $\Theta=\left[\theta_{i j}\right]$ denote $(m+p+1) \times n$ matrices with $(i, j)$ entries respectively given by $b_{i j}=\beta_{j-1}^{i-p-1}$ and $\theta_{i j}=\theta_{j-1}^{i-p-1}$. Then, denoting transposition by $T$, the extended Gram matrix involving $m$ antiderivatives and $p$ derivatives is given by

$$
G\left(f_{-m}, \ldots, f_{0}, \ldots, f_{p}\right)=(1 / 2) B \Theta^{T}
$$

Proof: Using eqn. 6 and the orthogonality property of eqn. 3, any entry $\left\langle f_{i}, f_{j}\right\rangle$ of $G$ is readily written as $\left\langle f_{i}, f_{j}\right\rangle=\sum_{k=0}^{n-1} \beta_{k}^{i} \beta_{k}^{j} /\left(2 \alpha_{k}\right)=\sum_{k=0}^{n-1} \beta_{k}^{i} \theta_{k}^{j} / 2$ by which eqn. 7 follows

\section{3 - Illustrative examples}

We first consider the transfer function given by Krajewski et al. in [6]

$$
F(s)=\frac{s^{2}+10 s+100}{1.21 s^{4}+3 s^{3}+110 s^{2}+230 s+100}
$$

with a view to deriving $G\left(f_{-1}, f_{0}, f_{1}\right)$ and a second-order reduced model. The entries in rows 2 of $B$ and $\Theta$ are readily obtained by the standard $\alpha-\beta$ Routh algorithm. A run of algorithm $D^{-}$yields the entries in rows 1 and a run of algorithm $D^{+}$yields the entries in rows 3: 


$$
\begin{aligned}
G=\frac{1}{2}\left[\begin{array}{cccc}
-2.026 & -0.076 & -0.174 & -0.403 \\
0.942 & 0.047 & 0.058 & 0.000 \\
-0.580 & 0.110 & 0.580 & 0.333
\end{array}\right]\left[\begin{array}{cccc}
-0.953 & -0.942 & -1.000 & -1.000 \\
0.443 & 0.580 & 0.333 & 0.000 \\
-0.273 & 1.354 & 3.333 & 0.826
\end{array}\right]^{T} \\
=\left[\begin{array}{cccc}
1.290 & -0.5 & -0.232 \\
-0.5 & 0.232 & 0 \\
-0.232 & 0 & 1.258
\end{array}\right]
\end{aligned}
$$

After the matrix product has been computed, the method described in [4] yields the $(1,-1,2)$ approximant whose second-order denominator matches exactly that of eqn. 44 in [6]. The squared $L_{2}$ norm of the error is equal to $1.125 \times 10^{-2}$ to be compared with $1.95 \times 10^{-2}$ obtained through balancing as pointed out in [6]. Note that the $L_{2}$ optimal value obtained via a Gauss-Newton nonlinear optimisation procedure is $1.099 \times 10^{-2}$.

In the case of the transfer function given by Hwang and Chen in [13],

$$
F(s)=\frac{9 s^{3}+42 s^{2}+31 s+10}{s^{4}+8 s^{3}+21 s^{2}+22 s+8}
$$

with a view to deriving $G\left(f_{0}, f_{1}, f_{2}\right)$ given by

$$
G=\left[\begin{array}{ccc}
11.40 & -40.50 & 111.7 \\
-40.50 & 158.2 & -450.0 \\
111.7 & -450.0 & 0.451
\end{array}\right]
$$

and a second order model as in the previous example, the technique described in [4] yields the $(0,1,2)$ approximant with an error norm of $1.5142 \times 10^{-2}$ while the approximant given by [13] yields $8.2904 \times 10^{-2}$.

The optimal $\mathrm{L}_{2}$ value in this case, calculated by Lucas in [14], is $7.0135 \times 10^{-3}$.

We finally refer to Pal's celebrated example [12]:

$$
F(s)=\frac{8 s^{2}+6 s+2}{s^{3}+4 s^{2}+5 s+2}
$$

with the corresponding Gram matrix $G$ :

$$
G=\left[\begin{array}{ccc}
0.451 & -0.125 & -1.194 \\
-0.125 & 0.694 & -0.5 \\
-1.194 & -0.5 & 9.222
\end{array}\right]
$$

This time we use Jain's model order reduction method [2] as described in [3] which yields an error norm of $3.007 \times 10^{-2}$ to be compared to Pal's original model yielding $4.098 \times 10^{-2}$. We may observe that our result is in fact very close to the optimal value obtained via Gauss-Newton optimisation providing $2.909 \times 10^{-2}$. 


\section{4 - Conclusion}

Efficient algorithms for orthogonal decomposition of derivatives and antiderivatives of functions with rational Laplace transforms have been presented. A simple method for computing the extended Gram matrix follows, whereby model order reduction very close to the optimal can be carried out without any optimising iterative procedure

\section{Acknowledgements}

The authors would like to acknowledge Brittany Region's financial support.

\section{References}

1 JAIN, V.K.: 'Decoupled method for approximation of signals by exponentials', IEEE Trans., 1970, SSC6, pp 244-246

2 JAIN, V.K., and GUPTA, R.D.: 'Identification of linear systems through a Grammian technique', Int. J. Control, 1970,12, (3), pp. 421-431

3 CALVEZ, L.C., VILBE, P., and BREHONNET, P.: 'Evaluation of scalar products of repeated integrals of a function with rational Laplace transform', Electron. Lett., 1988, 24, (11), pp.658-659

4 VILBE, P., CALVEZ, L.C., and SEVELLEC, M.: 'Suboptimal model reduction via least-square approximation of time-response by its derivatives and integrals', Electron. Lett., 1992, 28, (2), pp. 174175

5 SREEMAM, V., and AGATHOKLIS, P.: 'On the computation of the Gram matrix in time domain and its application', IEEE Trans., 1993, AC-38, (10), pp. 1516-1520

6 KRAJEWSKI, W., LEPSCHY, A., and VIARO, U.: 'Model reduction by matching Markov parameters, time moments, and impulse-response energies', IEEE Trans., 1995, AC-40, (5), pp. 949-953

7 LUCAS, TN.: 'Evaluation of scalar products of repeated integrals by Routh algorithm', Electron. Lett., 1988, 24, (20), pp.1290-1291

8 SREERAM, V., and GODDARD, P.: 'Evaluation of Gram matrix off-diagonal elements using system time moments', Electron. Lett., 1991, 27, (3), pp. 277-278

9 SREERAM, V., and AGATHOKLIS, P.: 'On the properties of Gram matrix', IEEE Trans, 1994, CAS I41, (3), pp. 234-237

10 HUTTON, M.F., and FRIEDLAND,B.: 'Routh approximations for reducing order of linear, timeinvariant systems', IEEE Trans., 1975, AC-20, (3), pp. 329-337

11 THERAPOS, C.P.: 'Balanced minimal realisation of SISO systems', Electron. Lett., 1983, 19, (11), pp. 424-426

12 PAL, J., 'Improved Padé approximants using stability equation method ', Electron. Lett., 1983, 19, (11), pp. 426-427

13 HWANG, C. and CHEN, M.-Y., A Multipoint Continued Fraction Expansion for Linear System Reduction, IEEE Trans. Automat. Contr., 1986, AC-31, pp. 648-651

14 LUCAS, T.N., J. Franklin Inst. 1993, vol.330, pp. 79-93 